Proceedings of the Edinburgh Mathematical Society (2005) 48, 365-373 (C)

DOI:10.1017/S0013091503000944 Printed in the United Kingdom

\title{
THREE POSITIVE SOLUTIONS FOR A CLASS OF ELLIPTIC SYSTEMS IN ANNULAR DOMAINS
}

\author{
JOÃO MARCOS DO Ó ${ }^{1}$, SEBASTIÁN LORCA ${ }^{2}$ AND PEDRO UBILLA ${ }^{3}$ \\ ${ }^{1}$ Departamento de Matemática, Universidade Federal da Paraíba, \\ 58059-900, João Pessoa, PB, Brazil (jmbo@mat.ufpb.br) \\ ${ }^{2}$ Departamento de Matemática, Universidad de Tarapacá, \\ Casilla 7-D, Arica, Chile (slorca@uta.cl) \\ ${ }^{3}$ Universidad de Santiago de Chile, Casilla 30\%, \\ Correo 2, Santiago, Chile (pubilla@usach.cl)
}

(Received 12 November 2003)

Abstract In this paper we study the existence and multiplicity of positive radial solutions for a class of semilinear elliptic systems in bounded annular domains with non-homogeneous boundary conditions by the use of a fixed-point theorem of cone expansion/compression type.

Keywords: elliptic systems; annular domains; positive radial solutions; multiplicity; fixed points

2000 Mathematics subject classification: Primary 35J65; 34B15; 34B18

\section{Introduction}

In this paper we study the existence and multiplicity of positive radial solutions for a class of semilinear elliptic systems in bounded annular domains. In fact, given real numbers $0<r_{1}<r_{2}$, we consider the system

$$
\left.\begin{array}{ll}
-\Delta u=a_{2} h(|x|, u, v) & \text { in } r_{1}<|x|<r_{2}, \\
-\Delta v=b_{2} k(|x|, u, v) & \text { in } r_{1}<|x|<r_{2}, \\
(u, v)=(0,0) & \text { on }|x|=r_{1}, \\
(u, v)=\left(a_{1}, b_{1}\right) & \text { on }|x|=r_{2},
\end{array}\right\}
$$

where $\left(a_{1}, b_{1}\right) \in[0,+\infty)^{2} \backslash\{(0,0)\},\left(a_{2}, b_{2}\right) \in(0,+\infty)^{2}$ and the nonlinearities $h$ and $k$ satisfy the following hypotheses.

$\left(\mathrm{H}_{0}\right) h, k:\left[r_{1}, r_{2}\right] \times[0,+\infty)^{2} \rightarrow[0,+\infty)$ are continuous functions such that there exist continuous functions, which are non-decreasing in the last two variables $h_{1}$ and $k_{1}$, satisfying

$$
\left.\begin{array}{l}
h_{1}(r, u, v) \leqslant h(r, u, v) \leqslant c_{h} h_{1}(r, u, v), \\
k_{1}(r, u, v) \leqslant k(r, u, v) \leqslant c_{k} k_{1}(r, u, v),
\end{array}\right\}
$$


where $c_{h}$ and $c_{k}$ are positive constants. We recall that $\ell$ is a non-decreasing function in the last two variables if $\ell\left(r, u_{1}, v_{1}\right) \leqslant \ell\left(r, u_{2}, v_{2}\right)$ whenever $u_{1} \leqslant u_{2}$ and $v_{2} \leqslant v_{2}$.

$\left(\mathrm{H}_{1}\right)$ There exist $\sigma_{1}, \sigma_{2}, \omega_{1}, \omega_{2} \in\left(r_{1}, r_{2}\right)$ with $\sigma_{1}<\sigma_{2}$ and $\omega_{1}<\omega_{2}$ such that

$$
h(r, u, v)>0 \quad \text { for each }(r, u, v) \in\left[\sigma_{1}, \sigma_{2}\right] \times\left([0,+\infty)^{2} \backslash\{(0,0)\}\right)
$$

or

$$
k(r, u, v)>0 \quad \text { for each }(r, u, v) \in\left[\omega_{1}, \omega_{2}\right] \times\left([0,+\infty)^{2} \backslash\{(0,0)\}\right) .
$$

Let us introduce the following notation:

$$
\ell_{0}:=\lim _{|(u, v)| \rightarrow 0} \frac{\ell(r, u, v)}{|(u, v)|} \text { and } \ell_{\infty}:=\lim _{|(u, v)| \rightarrow+\infty} \frac{\ell(r, u, v)}{|(u, v)|} .
$$

$\left(H_{2}\right) h_{0}=k_{0}=0$, uniformly in $\left[r_{1}, r_{2}\right]$ (superlinear at origin).

$\left(H_{3}\right) h_{\infty}=k_{\infty}=0$, uniformly in $\left[r_{1}, r_{2}\right]$ (sublinear at infinity).

We now state our main result.

Theorem 1.1. Assume that $h(r, u, v)$ and $k(r, u, v)$ satisfy $\left(\mathrm{H}_{0}\right)-\left(\mathrm{H}_{3}\right)$. Then the following hold.

(i) System (S) has at least one positive solution for all $\left(a_{1}, b_{1}\right) \in[0,+\infty)^{2} \backslash\{(0,0)\}$ and $\left(a_{2}, b_{2}\right) \in(0,+\infty)^{2}$.

(ii) There exists a positive constant $\Lambda>0$ such that, for all $\left(a_{2}, b_{2}\right) \in(0,+\infty)^{2}$ with $\min \left\{a_{2}, b_{2}\right\}>\Lambda$, there exists $\delta>0$ such that $(\mathrm{S})$ has at least three positive solutions for all $\left(a_{1}, b_{1}\right) \in[0,+\infty)^{2} \backslash\{(0,0)\}$ with $0<\left|\left(a_{1}, b_{1}\right)\right|<\delta$.

Our approach to prove Theorem 1.1 relies on fixed-point index theory. The following is a typical example of where our result may be applied:

$$
\left.\begin{array}{rlrl}
-\Delta u & =a_{2} c_{1}(|x|) \hat{h}(u, v) & & \text { in } r_{1}<|x|<r_{2}, \\
-\Delta v & =b_{2} c_{2}(|x|) \hat{k}(u, v) & & \text { in } r_{1}<|x|<r_{2}, \\
(u, v) & =(0,0) & & \text { on }|x|=r_{1}, \\
(u, v) & =\left(a_{1}, b_{1}\right) & & \text { on }|x|=r_{2},
\end{array}\right\}
$$

where $\hat{h}$ and $\hat{k}$ are continuous functions verifying $\hat{h}_{0}=\hat{k}_{0}=\hat{h}_{\infty}=\hat{k}_{\infty}=0$ and $c_{1}, c_{2}$ are non-negative and non-trivial functions. Moreover, we suppose the following two assumptions.

(i) $\hat{h}, \hat{k}:[0,+\infty)^{2} \rightarrow[0,+\infty)$ are continuous functions such that there exist nondecreasing continuous functions $h_{1}$ and $k_{1}$ such that

$$
\left.\begin{array}{l}
h_{1}(u, v) \leqslant \hat{h}(u, v) \leqslant \hat{c}_{h} h_{1}(u, v) \\
k_{1}(u, v) \leqslant \hat{k}(u, v) \leqslant \hat{c}_{k} k_{1}(u, v),
\end{array}\right\}
$$

where $\hat{c}_{h}$ and $\hat{c}_{k}$ are positive constants.

(ii) $\hat{h}(u, v)>0$ and $\hat{k}(u, v)>0$ for each $(u, v) \in\left([0,+\infty)^{2} \backslash\{(0,0)\}\right)$. 
Thus, according to the assumptions above on functions $\hat{h}$ and $\hat{k}$, it is not difficult to verify hypotheses $\left(\mathrm{H}_{0}\right)-\left(\mathrm{H}_{3}\right)$. Therefore, the conclusions of Theorem 1.1 are true.

The study of (1.4) is, in part, motivated by several recent results for elliptic boundaryvalue problems on annular domains. Among others, we mention $[\mathbf{1}, \mathbf{4 - 6}, \mathbf{8 - 1 0}]$ and the references therein. We refer to $[\mathbf{5}, \mathbf{6}]$ for homogeneous Dirichlet boundary conditions and [4], as well as [10], for non-homogeneous Dirichlet boundary conditions. In those articles is proved the existence of at least one or two positive solutions, while in the present paper we obtain multiplicity results that include the existence of at least three positive solutions. We would like to mention that we may apply Theorem 1.1 to more general classes of nonlinearities than those considered in (1.4). For example, we may consider in (S) nonlinearities like

$$
h(r, u, v)=\left(a(r)\left(u^{p_{1}}+v^{q_{1}}\right)+1\right) \Phi\left(b(r)\left(u^{p_{2}}+v^{q_{2}}\right)\right) \quad \text { and } \quad k(r, u, v)=\frac{(u+v)^{p_{3}}}{1+(u+v)^{q_{3}}},
$$

where $p_{1}, q_{1} \in(0,1), q_{2}, p_{2} \in(1,+\infty), q_{3}>p_{3}-1>0, a, b:\left[r_{1}, r_{2}\right] \rightarrow[0,+\infty)$ are continuous functions and $\Phi:[0,+\infty) \rightarrow[0,+\infty)$ is a non-decreasing continuous function satisfying

$$
\lim _{u \rightarrow 0} \frac{\Phi(u)}{u}=0 \quad \text { and } \quad \lim _{u \rightarrow+\infty} \frac{\Phi(u)}{u}=\hat{c}>0 .
$$

We notice that the functions $a, b$ may vanish in parts of the interval $\left[r_{1}, r_{2}\right]$. Finally, we observe that hypothesis $\left(\mathrm{H}_{1}\right)$ allows us to take nonlinearities $h$ and $k$ without the monotonicity property.

The rest of this paper is organized as follows. Section 2 contains preliminary results and $\S 3$ is devoted to proving our main result, Theorem 1.1.

\section{Preliminary results}

We give some results which will be necessary to prove Theorem 1.1 in the next section.

The proof of Theorem 1.1 relies on fixed-point index theory in the frame of the ordinarydifferential-equation (ODE) technique. Since we are interested in the existence of radial solutions, by applying consecutive changes of variables with $r=|x|$ and

$$
t=-\eta_{1} r^{2-N}+\eta_{2}, \quad \text { where } \eta_{1}=\frac{\left(r_{1} r_{2}\right)^{N-2}}{r_{2}^{N-2}-r_{1}^{N-2}} \text { and } \eta_{2}=\frac{r_{2}^{N-2}}{r_{2}^{N-2}-r_{1}^{N-2}}
$$

we can transform (S) into the following system of second-order ODEs,

$$
\left.\begin{array}{ll}
-u^{\prime \prime}=a_{2} f\left(t, u, v, a_{1}, b_{1}\right) & \text { in }(0,1), \\
-v^{\prime \prime}=b_{2} g\left(t, u, v, a_{1}, b_{1}\right) & \text { in }(0,1), \\
u(0)=u(1)=0, & \\
v(0)=v(1)=0, &
\end{array}\right\}
$$


where here the nonlinearities $f$ and $g$ are given by

$$
\left.\begin{array}{c}
f\left(t, u, v, a_{1}, b_{1}\right)=d(t) h\left(\left(\frac{\eta_{1}}{\eta_{2}-t}\right)^{1 /(N-2)}, u+t a_{1}, v+t b_{1}\right) \\
g\left(t, u, v, a_{1}, b_{1}\right)=d(t) k\left(\left(\frac{\eta_{1}}{\eta_{2}-t}\right)^{1 /(N-2)}, u+t a_{1}, v+t b_{1}\right), \\
d(t)=(1-N)^{2} \frac{\eta_{1}^{2 /(N-2)}}{\left(\eta_{2}-t\right)^{2(N-1) /(N-2)}} .
\end{array}\right\}
$$

Setting

$$
\begin{aligned}
& f_{1}\left(t, u, v, a_{1}, b_{1}\right)=d(t) h_{1}\left(\left(\frac{\eta_{1}}{\eta_{2}-t}\right)^{1 /(N-2)}, u+t a_{1}, v+t b_{1}\right), \\
& g_{1}\left(t, u, v, a_{1}, b_{1}\right)=d(t) k_{1}\left(\left(\frac{\eta_{1}}{\eta_{2}-t}\right)^{1 /(N-2)}, u+t a_{1}, v+t b_{1}\right),
\end{aligned}
$$

we observe that, from (1.1) and (2.1),

$$
\left.\begin{array}{l}
f_{1}\left(t, u, v, a_{1}, b_{1}\right) \leqslant f\left(t, u, v, a_{1}, b_{1}\right) \leqslant c_{h} f_{1}\left(t, u, v, a_{1}, b_{1}\right) \\
g_{1}\left(t, u, v, a_{1}, b_{1}\right) \leqslant k\left(t, u, v, a_{1}, b_{1}\right) \leqslant c_{k} g_{1}\left(t, u, v, a_{1}, b_{1}\right) .
\end{array}\right\}
$$

It is not difficult to show that if the pair $(u, v)$ is a solution of $(\mathrm{R})$, then, for all $t \in[0,1]$,

$$
\left.\begin{array}{l}
u(t)=a_{2} \int_{0}^{1} K(t, \tau) f\left(\tau, u(\tau), v(\tau), a_{1}, b_{1}\right) \mathrm{d} \tau \\
v(t)=b_{2} \int_{0}^{1} K(t, \tau) g\left(\tau, u(\tau), v(\tau), a_{1}, b_{1}\right) \mathrm{d} \tau
\end{array}\right\}
$$

where $K(t, \tau)$ is Green's function

$$
K(t, s):= \begin{cases}t(1-s) & \text { if } t \leqslant s \\ s(1-t) & \text { if } t>s\end{cases}
$$

Let

$$
\begin{aligned}
A(u, v)(t) & :=a_{2} \int_{0}^{1} K(t, \tau) f\left(\tau, u(\tau), v(\tau), a_{1}, b_{1}\right) \mathrm{d} \tau \\
B(u, v)(t) & :=b_{2} \int_{0}^{1} K(t, \tau) g\left(\tau, u(\tau), v(\tau), a_{1}, b_{1}\right) \mathrm{d} \tau \\
F(u, v) & :=(A(u, v), B(u, v)) .
\end{aligned}
$$

Therefore, system (2.3) is equivalent to the fixed-point equation

$$
F(u, v)=(u, v)
$$


in the usual Banach space

$$
X=C([0,1], \mathbb{R}) \times C([0,1], \mathbb{R})
$$

endowed with the norm $\|(u, v)\|:=\|u\|_{\infty}+\|v\|_{\infty}$, where $\|w\|_{\infty}:=\sup _{t \in[0,1]}|w(t)|$.

In order to verify the existence of positive solutions for (S), we introduce now the following fixed-point theorem of cone expansion/compression type. We refer to $[\mathbf{2}, \mathbf{3}, \mathbf{7}]$ for proofs and further discussion of the fixed-point theory.

Lemma 2.1. Let $X$ be a Banach space with norm $|\cdot|$ and let $C \subset X$ be a cone in $X$. For $R>0$, define $C_{R}=C \cap B[0, R]$, where $B[0, R]=\{x \in X:|x| \leqslant R\}$ denotes the closed ball of radius $R$ centred at origin $X$. Assume that $F: C_{R} \rightarrow C$ is a compact map and that there exists $0<r<R$ such that

$$
|F x| \leqslant|x|, \quad x \in \partial C_{r} \quad \text { and } \quad|F x| \geqslant|x|, \quad x \in \partial C_{R},
$$

or

$$
|F x| \geqslant|x|, \quad x \in \partial C_{r} \quad \text { and } \quad|F x| \leqslant|x|, \quad x \in \partial C_{R},
$$

where $\partial C_{R}=\{x \in C:|x|=R\}$. Then $F$ has a fixed point $u \in C$ with $r<|u|<R$.

Let us consider the cone $C$ in $X$ defined by

$$
C=\{(u, v) \in X:(u, v)(0)=(u, v)(1)=0 \text { and } u, v \text { are concave functions }\} .
$$

Using the concavity of the functions $u(t)$ and $v(t)$, we may easily prove the following elementary result.

Lemma 2.2. For each $(u, v) \in C$ and $(\alpha, \beta) \subset(0,1)$, we have

$$
\inf _{t \in[\alpha, \beta]}(u(t)+v(t)) \geqslant \alpha(1-\beta)\|(u, v)\| .
$$

Lemma 2.3. $F: X \rightarrow X$ is completely continuous and $F(C) \subset C$.

Proof. We only give the main ideas of the proof. The Arzela-Ascoli theorem implies that $A$ and $B$ are concave functions and therefore $F: X \rightarrow X$ is completely continuous. It is easy to see that $A$ and $B$ (the coordinates functions of $F(u, v)$ ) are twice differentiable on $(0,1)$ with $A^{\prime \prime} \leqslant 0$ and $B^{\prime \prime} \leqslant 0$. This implies that $A$ and $B$ are concave functions and therefore $F(C) \subset C$.

\section{Proof of Theorem 1.1}

\subsection{The existence of the first positive solution}

Using the fact that $f, g$ satisfy assumptions $\left(\mathrm{H}_{0}\right),\left(\mathrm{H}_{1}\right)$ and $\left(\mathrm{H}_{3}\right)$, we apply Lemma 2.1 to prove the existence of a first positive solution for (S). Let $\left(a_{1}, b_{1}\right) \in[0,+\infty)^{2} \backslash\{(0,0)\}$ and $\left(a_{2}, b_{2}\right) \in(0,+\infty)^{2}$ be fixed. 
Lemma 3.1. Assume that conditions $\left(\mathrm{H}_{0}\right)$ and $\left(\mathrm{H}_{1}\right)$ hold. Then there exists $R_{1}>0$ such that, for all $R \in\left(0, R_{1}\right]$, we have

$$
\|F(u, v)\| \geqslant\|(u, v)\| \quad \text { for each }(u, v) \in \partial C_{R}
$$

Proof. From $\left(\mathrm{H}_{0}\right)$, we have

$$
\begin{aligned}
f\left(t, u, v, a_{1}, b_{1}\right) & =d(t) h\left(\left(\frac{\eta_{1}}{\eta_{2}-t}\right)^{1 /(N-2)}, u+t a_{1}, v+t b_{1}\right) \\
& \geqslant d(t) h_{1}\left(\left(\frac{\eta_{1}}{\eta_{2}-t}\right)^{1 /(N-2)}, t a_{1}, t b_{1}\right) .
\end{aligned}
$$

Thus, assuming (1.2), we obtain that there exist constants $0<\alpha_{1}<\beta_{1}<1$ such that

$$
f_{0}=\lim _{|(u, v)| \rightarrow 0} \frac{f\left(t, u, v, a_{1}, b_{1}\right)}{|(u, v)|}=+\infty \quad \text { uniformly for } t \in\left[\alpha_{1}, \beta_{1}\right] .
$$

Now, using (3.1), for each $M>0$, there exists $R_{1}>0$ such that

$$
f\left(t, u, v, a_{1}, b_{1}\right) \geqslant M|(u, v)| \text { for all }(u, v) \in\left[0, R_{1}\right]^{2} .
$$

Therefore, for all $(u, v) \in C_{R_{1}}$,

$$
\begin{aligned}
\|F(u, v)\| & \geqslant\|A(u, v)\|_{\infty} \\
& \geqslant a_{2} \int_{0}^{1} K\left(\frac{1}{2}, \tau\right) f\left(\tau, u(\tau), v(\tau), a_{1}, b_{1}\right) \mathrm{d} \tau \\
& \geqslant a_{2} M \int_{\alpha_{1}}^{\beta_{1}} K\left(\frac{1}{2}, \tau\right)[u(\tau)+v(\tau)] \mathrm{d} \tau \\
& \geqslant a_{2} \alpha_{1}\left(1-\beta_{1}\right) M\|(u, v)\| \int_{\alpha_{1}}^{\beta_{1}} K\left(\frac{1}{2}, \tau\right) \mathrm{d} \tau .
\end{aligned}
$$

Finally, taking $M>0$ sufficiently large, we conclude the proof of Lemma 3.1.

Lemma 3.2. Assume hypotheses $\left(\mathrm{H}_{0}\right)$ and $\left(\mathrm{H}_{3}\right)$. Then there exists $R_{2}>R_{1}$ such that, for all $R \geqslant R_{2}$, we have

$$
\|F(u, v)\| \leqslant\|(u, v)\| \quad \text { for each }(u, v) \in \partial C_{R} .
$$

Proof. From assumptions $\left(\mathrm{H}_{0}\right)$ and $\left(\mathrm{H}_{3}\right)$, it is not difficult to see that

$$
\lim _{|(u, v)| \rightarrow+\infty} \frac{f_{1}\left(r, u, v, a_{1}, b_{1}\right)}{|(u, v)|}=\lim _{|(u, v)| \rightarrow+\infty} \frac{g_{1}\left(r, u, v, a_{1}, b_{1}\right)}{|(u, v)|}=0 .
$$

Thus, given $\delta>0$, there exists $R_{2}>R_{1}$ such that, for all $\tau \in[0,1]$ and $|(u, v)| \geqslant R_{2}$,

$$
f_{1}\left(\tau, u, v, a_{1}, b_{1}\right) \leqslant \delta|(u, v)| \quad \text { and } \quad g_{1}\left(\tau, u, v, a_{1}, b_{1}\right) \leqslant \delta|(u, v)| .
$$


Thus, using (2.2), for all $(u, v) \in C_{R_{2}}$,

$$
\begin{aligned}
\|A(u, v)\|_{\infty} & =a_{2} \int_{0}^{1} K(t, \tau) f\left(\tau, u(\tau), v(\tau), a_{1}, b_{1}\right) \mathrm{d} \tau \\
& \leqslant a_{2} c_{h} \int_{0}^{1} K(t, \tau) f_{1}\left(\tau, u(\tau), v(\tau), a_{1}, b_{1}\right) \mathrm{d} \tau \\
& \leqslant a_{2} c_{h} \int_{0}^{1} K(t, \tau) f_{1}\left(\tau,\|u\|_{\infty},\|v\|_{\infty}, a_{1}, b_{1}\right) \mathrm{d} \tau \\
& \leqslant a_{2} c_{h} \delta\|(u, v)\| \int_{0}^{1} K(t, \tau) \mathrm{d} \tau .
\end{aligned}
$$

Similarly, we may prove that

$$
\|B(u, v)\|_{\infty} \leqslant a_{2} c_{h} \delta\|(u, v)\| \int_{0}^{1} K(t, \tau) \mathrm{d} \tau .
$$

Hence, taking $\delta>0$ small enough, we have

$$
\|F(u, v)\|=\|A(u, v)\|_{\infty}+\|B(u, v)\|_{\infty} \leqslant R_{2}=\|(u, v)\| .
$$

In view of Lemmas 3.1 and 3.2, as a direct consequence of Lemma 2.1, since $R_{1}<R_{2}$, the proof of the first part of Theorem 1.1 is now complete.

\subsection{The proof of the second part of Theorem 1.1}

Lemma 3.3. Assume that conditions $\left(\mathrm{H}_{0}\right)$ and $\left(\mathrm{H}_{1}\right)$ hold. Given $\bar{R}>0$, there exists $\Lambda>0$ such that, for all $\left(a_{1}, b_{1}\right) \in[0,+\infty)^{2}$ and $\left(a_{2}, b_{2}\right) \in(0,+\infty)^{2}$ with $\min \left\{a_{2}, b_{2}\right\} \geqslant \Lambda$, we have

$$
\|F(u, v)\| \geqslant\|(u, v)\| \quad \text { for each }(u, v) \in \partial C_{\bar{R}},
$$

where the constant $\Lambda$ is independent of the parameters $a_{1}$ and $b_{1}$.

Proof. We assume that (1.2) holds. Let $\alpha_{1}, \beta_{1} \in(0,1)$, as in the proof of Lemma 3.1, $\bar{R}>0$ and $(u, v) \in C_{\bar{R}}$. According to assumption $\left(\mathrm{H}_{0}\right)$ and Lemma 2.2, we have

$$
\begin{aligned}
\|A(u, v)\|_{\infty} & \geqslant a_{2} \int_{\alpha_{1}}^{\beta_{1}} K\left(\frac{1}{2}, \tau\right) f\left(\tau, u(\tau), v(\tau), a_{1}, b_{1}\right) \mathrm{d} \tau \\
& \geqslant a_{2} \int_{\alpha_{1}}^{\beta_{1}} K\left(\frac{1}{2}, \tau\right) f_{1}\left(\tau, u(\tau), v(\tau), a_{1}, b_{1}\right) \mathrm{d} \tau \\
& \geqslant a_{2} \int_{\alpha_{1}}^{\beta_{1}} K\left(\frac{1}{2}, \tau\right) f_{1}\left(\tau, \alpha_{1}\left(1-\beta_{1}\right)\|u\|_{\infty}, \alpha_{1}\left(1-\beta_{1}\right)\|v\|_{\infty}, 0,0\right) \mathrm{d} \tau .
\end{aligned}
$$

According to assumption (1.2), we see that

$$
\bar{C}:=\min \left\{f_{1}\left(\tau, \alpha_{1}\left(1-\beta_{1}\right) u, \alpha_{1}\left(1-\beta_{1}\right) v, 0,0\right): \tau \in\left[\alpha_{1}, \beta_{1}\right], u+v=\bar{R}\right\}>0 .
$$


Thus we obtain that there exists a constant $\tilde{a}>0$ such that

$$
\|A(u, v)\|_{\infty} \geqslant a_{2} \tilde{a} .
$$

From (3.3), we get

$$
\|F(u, v)\| \geqslant a_{2} \tilde{a} \geqslant a_{2} \frac{\tilde{a}}{\bar{R}} \bar{R} .
$$

Hence there exists a positive constant $\Lambda$ such that, for all $\left(a_{1}, b_{1}\right) \in[0,+\infty)^{2}$ and $\left(a_{2}, b_{2}\right) \in(0,+\infty)^{2}$ with $\min \left\{a_{2}, b_{2}\right\} \geqslant \Lambda$, we have

$$
\|F(u, v)\| \geqslant\|(u, v)\| \quad \text { for each }(u, v) \in \partial C_{\bar{R}} .
$$

We notice that $\Lambda$ does not depend on the parameters $a_{1}, b_{1}$.

Lemma 3.4. Assume that $\left(\mathrm{H}_{0}\right)$ and $\left(\mathrm{H}_{2}\right)$ hold. Given $\left(a_{2}, b_{2}\right) \in(0,+\infty)^{2}$ with $\min \left\{a_{2}, b_{2}\right\} \geqslant \Lambda$, there exists $R_{2} \in(0, \bar{R})$ and $\delta_{1}>0$ such that, for all $\left(a_{1}, b_{1}\right) \in[0,+\infty)^{2}$, with $a_{1}+b_{1}<\delta_{1}$, we have

$$
\|F(u, v)\| \leqslant\|(u, v)\| \quad \text { for each }(u, v) \in \partial C_{R_{2}} .
$$

Proof. Using assumptions $\left(\mathrm{H}_{0}\right)-\left(\mathrm{H}_{2}\right)$, we have

$$
\lim _{\left|\left(u, v, a_{1}, b_{1}\right)\right| \rightarrow 0} \frac{f\left(t, u, v, a_{1}, b_{1}\right)}{\left|\left(u, v, a_{1}, b_{1}\right)\right|}=0 .
$$

Thus, given $\varepsilon>0$, there exists $R_{2} \in(0, \bar{R})$ such that, for all $\left(u, v, a_{1}, b_{1}\right)$ with $u+v \leqslant R_{2}$ and $a_{1}+b_{1} \leqslant R_{2}$, we get

$$
f\left(t, u, v, a_{1}, b_{1}\right) \leqslant \varepsilon\left|\left(u, v, a_{1}, b_{1}\right)\right| .
$$

Let $(u, v) \in C_{R_{2}}$ and $a_{1}+b_{1} \leqslant R_{2}$. From the above estimate, we have

$$
\begin{aligned}
A(u, v)(t) & =a_{2} \int_{0}^{1} K(t, \tau) f(\tau, u(\tau), v(\tau)) \mathrm{d} \tau \\
& \leqslant c_{h} a_{2} \int_{0}^{1} K(t, \tau) f_{1}(\tau, u(\tau), v(\tau)) \mathrm{d} \tau \\
& \leqslant \varepsilon c_{h} a_{2}\left(\|(u, v)\|+a_{1}+b_{1}\right) \int_{0}^{1} K(t, \tau) \mathrm{d} \tau \\
& \leqslant \varepsilon c_{h} a_{2} 2 R_{2} \int_{0}^{1} K(t, \tau) \mathrm{d} \tau .
\end{aligned}
$$

Taking $\varepsilon>0$ sufficiently small, we have

$$
\|A(u, v)\|_{\infty} \leqslant \frac{1}{2}\|(u, v)\| \text { for each }(u, v) \in \partial C_{R_{2}} .
$$

We can now proceed analogously to prove that

$$
\|B(u, v)\|_{\infty} \leqslant \frac{1}{2}\|(u, v)\| \text { for each }(u, v) \in \partial C_{R_{2}} .
$$

These two estimates together prove (3.5). 
Finally, by considering $\bar{R}>R_{2}$ given in Lemmas 3.3 and 3.4, respectively, we apply once again Lemmas 3.1 and 3.2 to obtain $R_{3}$ and $R_{4}$, with $0<R_{3}<R_{2}<\bar{R}<R_{4}$, such that

$$
\begin{array}{ll}
\|F(u, v)\| \geqslant\|(u, v)\|, & (u, v) \in \partial C_{R_{3}}, \\
\|F(u, v)\| \leqslant\|(u, v)\|, & (u, v) \in \partial C_{R_{2}}, \\
\|F(u, v)\| \geqslant\|(u, v)\|, & (u, v) \in \partial C_{\bar{R}}, \\
\|F(u, v)\| \leqslant\|(u, v)\|, & (u, v) \in \partial C_{R_{4}} .
\end{array}
$$

Therefore, we can apply Lemma 2.1 to get three fixed points of $F$ in $C$ satisfying

$$
R_{3}<\left\|\left(u_{1}, v_{1}\right)\right\|<R_{2}<\left\|\left(u_{2}, v_{2}\right)\right\|<\bar{R}<\left\|\left(u_{3}, v_{3}\right)\right\|<R_{4},
$$

and the proof is complete.

Acknowledgements. J.M.Ó. was supported by CNPq, PRONEX-MCT/Brazil and Millennium Institute for the Global Advancement of Brazilian Mathematics-IM-AGIMB. S.L. was supported by UTA grant 4734-02. P.U. was supported by DICYT-USACH.

Part of this work was done while the authors were visiting IMECC-UNICAMP/ BRAZIL. The authors thank Professor Djairo de Figueiredo and all the faculty and staff of IMECC-UNICAMP for their kind hospitality.

\section{References}

1. C. Bandle And L. A. Peletier, Nonlinear elliptic problems with critical exponent in shrinking annuli, Math. Ann. 280 (1988), 1-19.

2. D. G. DE Figueiredo, Positive solutions of semilinear elliptic equations, Lecture Notes in Mathematics, vol. 957, pp. 34-87 (Springer, 1982).

3. K. Deimling, Nonlinear functional analysis (Springer, 1985).

4. J. M. DO Ó, S. LoRCA AND P. UBILla, Local superlinearity for elliptic systems involving parameters, J. Diff. Eqns, in press.

5. D. R. Dunninger And H. WAng, Existence and multiplicity of positive solutions for elliptic systems, Nonlin. Analysis 29 (1997), 1051-1060.

6. D. R. DUNNINGER AND H. WANG, Multiplicity of positive radial solutions for an elliptic system on an annulus, Nonlin. Analysis 42 (2000), 803-811.

7. D. Guo and V. Lakshmikantham, Nonlinear problem in abstract cones (Academic, 1988).

8. D. D. HAI, Positive solutions for semilinear elliptic equations in annular domains, Nonlin. Analysis 37 (1999), 1051-1058.

9. M. G. LeE AND S. S. Lin, On the positive solution for semilinear elliptic equations on annular domain with non-homogeneous Dirichlet boundary conditions, J. Math. Analysis Applic. 181 (1994), 348-361.

10. Y.-H. LEE, Multiplicity of positive radial solutions for multiparameter semilinear elliptic systems on an annulus, J. Diff. Eqns 174 (2001), 420-441. 